\title{
Study on the mechanism and application of surface eddy current damper
}

\author{
Chen Haiyan \\ Nanchang Institute of Science \& Technology, Nanchang 330108, China
}

\begin{abstract}
Key words: eddy current damper; surface structure; mechanical vibration; precision machine; damping force
\end{abstract}

\begin{abstract}
In the case of a thin surface precision mechanical device, it is often accompanied by mechanical vibration, mechanical vibration will reduce the precision of the device. In order to eliminate the influence of the vibration, the surface eddy current damper is introduced, which can quickly generate the damping force of multi degree of freedom, and has the advantages of simple structure, high safety and reliability. The mechanism of the eddy current damper is analyzed, and based on the calculation and analysis of the electromagnetic damping force, the vibration suppression effect of the eddy current damper is designed. The experimental results show that the surface mechanical device of precision parts vibration when using eddy current damper, vibration suppression of surface mechanical device type disk can be effective, the fast response speed, high reliability and safety, which has important reference value for the study of vibration reduction of panel type precision machinery parts.
\end{abstract}

\section{Introduction}

The relative motion between the conductor and the magnetic field will produce eddy current, and the magnetic field generated by the eddy current is opposite to the polarity of the original magnetic field, resulting in a force that hinders the relative motion, this phenomenon is called electromagnetic damping. The eddy current damper can effectively reduce the kinetic energy of the mechanical system and restrain the vibration of the mechanical device, in particular the precision mechanical device. The eddy current damper adopts the mechanism of non contact damping force, and can work safely and stably without any conditions, its production cost is low. Compared with the general damper, the eddy current damper is not disturbed by the external conditions, which increases the reliability of the system.

\section{Mechanism and overall design of surface eddy current damper}

For the design of surface eddy current damper, it is necessary to understand the mechanism of eddy current. As shown in Figure 1, in a static magnetic field, when the conductor plate moves at a certain speed, the flux in the surface conductor plate will change, and the induced current will be generated, the induced current is eddy current. The eddy current will re create a magnetic field, the magnetic field and static electromagnetic field instead, thus impeding the magnet and the plane conductor plate movement, which will produce eddy current damping force. 


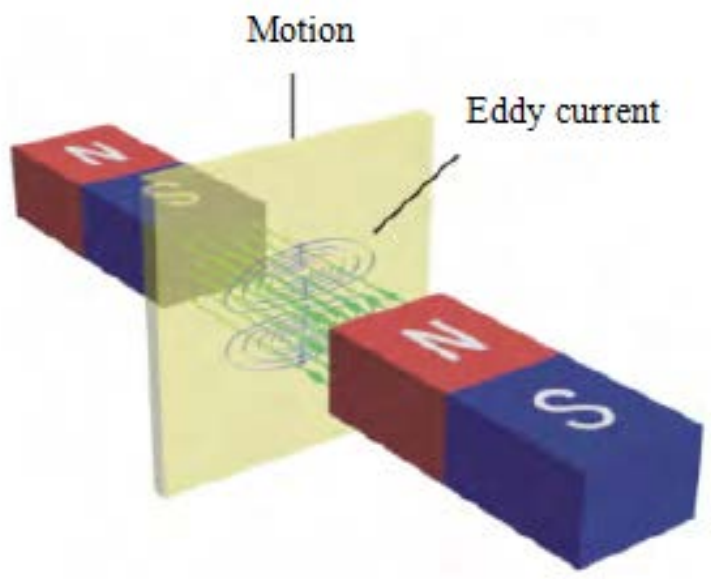

Fig.1 Eddy current damping mechanism

According to the basic principle of eddy current damping force, the surface damper is designed, the purpose of the damper is to reduce the vibration of the surface device. Surface disc of surface damper is fixed on the motor, the damper is wrapped around the inner surface of the silicon steel sheet to form a magnetic pole, power will be formed after the electromagnetic field, it is driven by the power driver module. There is a certain gap between the damper and the interview panel, and no contact between the damper, so it will not produce mechanical wear, the principle of the structure is shown in Figure 2.

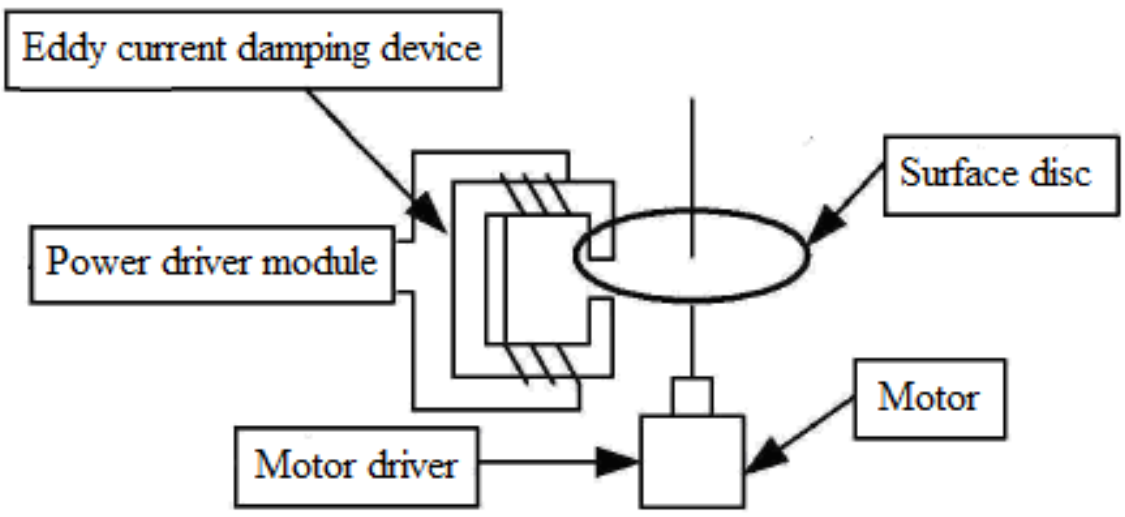

Fig.2 Schematic diagram of surface eddy current damper

As shown in Figure 2, when the damper is energized, the electromagnetic field will be generated in the air gap, the surface disc will produce eddy current in the process of rotation, when the disc vibrates, the gap between the panel and the eddy current damper changes, resulting in an electromagnetic damping force. The damping force can restrain the vibration of the disc, so that the device can reach a stable working state, eliminate the damage caused by the vibration to the device, and improve the operation quality and operation efficiency of the device.

\section{Calculation and analysis of electromagnetic damping force of surface eddy current damper}

The surface damper is composed of two sets of one-dimensional permanent magnet arrays, which are orthogonal to each other, each of which is composed of a number of permanent magnet arrays. In the calculation, we can calculate the electromagnetic damping force by changing the current of the conductor plate, and conductor current density can be written as Formula (1),

$$
J=\sigma(v \times B)
$$


In Formula (1), $\sigma$ is electrical conductivity of planar plates, $v$ is relative velocity between plane and magnetic field, $B$ is magnetic flux density, the eddy current damping can be expressed as

$$
F=\int_{V}(J \times B) d V
$$

In order to simplify the analysis, Assuming that the flux density along $x, y, z$, is similar, the electromagnetic damping can be written in the $x$ direction, its expression is:

$$
F_{x}=\sigma v_{x} C l_{y} \int_{-N x \tau / 4}^{N x \tau / 4} B^{2} d x
$$

In Formula (2), $v_{x}$ is the velocity of the $x$ direction, $c$ is the thickness of the surface conductor plate, $l_{y}$ is the effective length in the $y$ direction of the planar conductor plate, $N_{x}$ is the number of permanent magnets contained in the permanent magnet array in the $x$ direction. Similarly, it is assumed that the magnetic flux density along the $x, y$ and $z$ directions is similar, the magnetic flux density can be written as an electromagnetic damping in the $y$ direction, its expression is Formula (4).

$$
F_{x}=\sigma v_{x} C l_{x} \int_{-N y \tau / 4}^{N y \tau / 4} B^{2} d y
$$

In Formula (4), $v_{y}$ is the velocity of the $y$ direction, $c$ is the thickness of surface conductor plate, $l_{x}$ is the effective length in the $x$ direction of surface conductor plate, $N_{y}$ is is the number of permanent magnets contained in the permanent magnet array in the $y$ direction.

Damping coefficient must be considered when calculating damping force, and damping coefficient can be considered as the ratio of damping force and velocity, and the expression can be written as Formula (5).

$$
D=F / v
$$

By controlling the magnitude of the damping force, the vibration of the mechanism can be controlled, and the next section will be focused on.

\section{Performance analysis of surface mounted eddy current damper}

In order to verify the surface elimination effect of mechanical vibration damper, the experiment system is designed to control the disk vibration damper, which includes vibration, vibration signal detection, damping force driving environment, the main experimental process as shown in Figure 3.

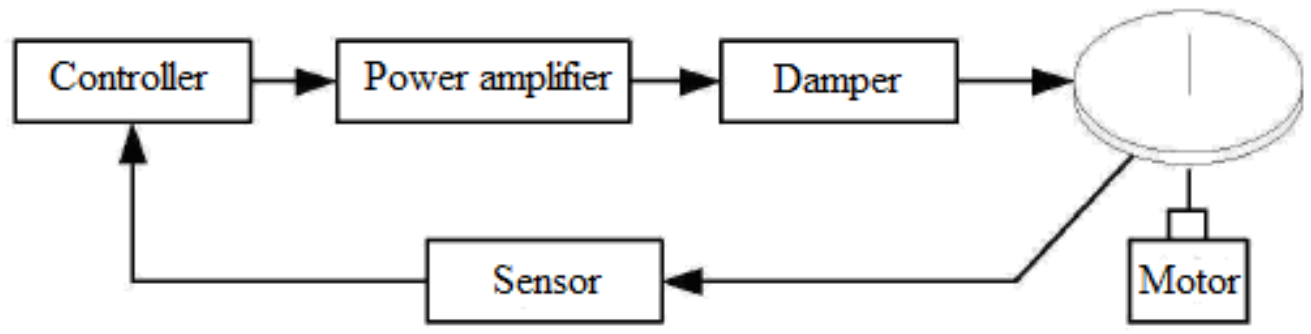

Fig. 3 The principle of experimental test process

As shown in Figure 3, the use of single-chip drive stepper motor in the experimental process, the disc starts to rotate in the rotating process because of its low mass will produce vibration, in order to 
verify the eddy current damper were collected on the vibration information, the vibration signal as shown in Figure 4.

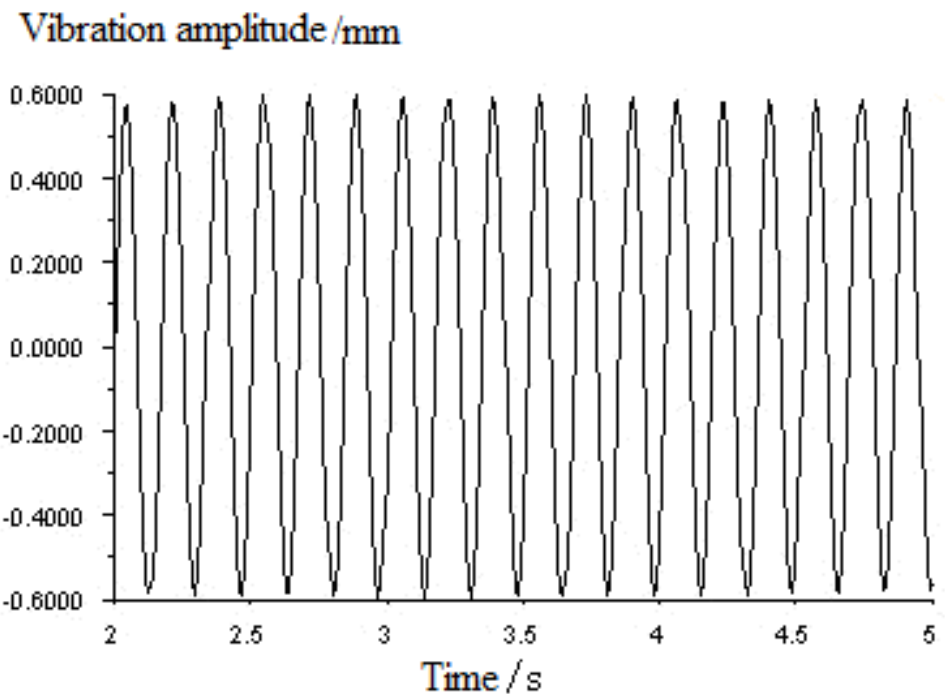

Fig. 4 The acquisition drawing of vibration signal

As shown in Figure 4, the displacement curves of disk structure generated during vibration is obtained through vibration signal acquisition, experimental device power will produce eddy current, thereby suppressing the generation of vibration on the disc, and the inhibitory effects are shown in table 1.

Table 1 The vibration reduction scale

\begin{tabular}{|c|c|c|}
\hline $\begin{array}{c}\text { Experimental } \\
\text { disk point }\end{array}$ & $\begin{array}{c}\text { Maximum amplitude of vibration } \\
\text { before the eddy current damper (mm) }\end{array}$ & $\begin{array}{c}\text { Maximum amplitude of vibration } \\
\text { after the eddy current damper (mm) }\end{array}$ \\
\hline 1 & 0.61 & 0.32 \\
\hline 2 & 0.56 & 0.28 \\
\hline 3 & 0.42 & 0.20 \\
\hline 4 & 0.33 & 0.13 \\
\hline 5 & 0.26 & 0.12 \\
\hline 6 & 0.18 & 0.08 \\
\hline
\end{tabular}

As shown in Table 1, the vibration signals of the disc vibration with and without eddy current damper are collected, from the statistical results, it can be found that the vibration of the disk has been greatly reduced by the inhibition of the eddy current damper, thus verifying the reliability of the electric damping eddy current device.

\section{Conclusion}

In order to restrain the vibration of the surface mechanical parts with higher precision, to improve the operating accuracy of the mechanical devices, an eddy current damper is introduced in this paper. Through the calculation and analysis of electromagnetic damping force, the mechanism of the eddy current damper is studied, and the structure of the damper is designed. Finally, the suppression effect of the damper is studied by experimental method. The experimental results show that the inhibitory effect of eddy current damper disk vibration can be greatly reduced, which verified the inhibitory effect of eddy current damper vibration type opposite precision machinery parts, is a kind of high efficient damper is safe and reliable, simple structure and low price. 


\section{Acknowledgement}

The work was supported by the science and technology project of Jiangxi Provincial Education Department with the project number GJJ161226 and the project name Research on the Mechanism and Application of Surface Eddy Current Damper.

\section{Reference}

[1] Kou Baoquan, Jin Yinxi, Zhang He, et al. Development and application prospect of electromagnetic damper [J]. Proceedings of the CSEE, Vol.35 (12), (2015), p.3132-3143

[2] Chen Zhengqing, Huang Zhiwen, Wang Jianhui, et al. Basic requirements and eddy currents of TMD for bridges TMD[J]. Journal of Hunan University(Natural Sciences), Vol.40 (8), (2013), p.6-10

[3] Wang Zhihao, Hua Xugang, Chen Zhengqing, et al. Micro permanent electric magnet type eddy current damping TMD footbridge vibration model test research of [J]. Journal of Vibration and Shock, Vol.33 (20) , (2014), p.129-132

[4] Wang Zhihao, Chen Zhengqing. Study on vibration control of stay cables by passive electromagnetic damper [J]. Journal of Vibration and Shock, Vol.33 (9), (2014), p.94-99

[5] Wang Youlin, Liu Jinglin. Design and research of electromagnetic damper [J]. Journal of Northwestern Polytechnical University, Vol.24 (3), (2006), p.358-362.

[6] Wang Youlin, Liu Jinglin, Ding Chong. Calculation and analysis of eddy current in electromagnetic damper [J]. Micromotor, Vol.39 (2), (2006), p.29-31.

[7] Yin Huajie, Ho Feng. Equivalent model of permanent magnet for permanent magnet motor [J]. Micromotor, Vol.37 (6), (2004), p.14-18.

[8] Zhang Bowen, Wang Xiaoyong, Hu Yongli. Integrated analysis of vibration effects on imaging of high resolution space camera[J], Spacecraft Recovery \& Remote Sensing,Vol.33 (2), (2012), p.60-66.

[9] Cao Qingsong, Zhang Min. Calculation and analysis of damping force of transverse eddy current damper [J]. Noise and Vibration Control, No.05, (2010) , p.181-185.

[10] Cao Qingsong, Wang Shunfa. Research progress of active vibration control technology based on electromagnetic damper [J]. Noise and Vibration Control, No.03 (2008), p. 1-5.

[11] H. Sodano, J. Bae. Eddy current damping in structures[J]. Shock and Vibration Digest, Vol.36(6), (2004), p.469.

[12] D. Kienholz, C. Smith, W. Haile. Magnetically damped vi-bration isolation system for a space shuttle payload[J]. In-ternational Society for Optics and Photonics, (1996), p.272-280.

[13] B. Ebrahimi, M. Khamesee, F. Golnaraghi. Permanentmagnet configuration in design of an eddy current damper[J]. Microsystem Technologies, Vol.16(1-2), (2010), p.19-24.

[14] L. Zuo, S. Nayfeh, X. Chen. Design and analysis of a newtype of electromagnetic damper with increased energy den-sity[J]. Journal of Vibration and Acoustics, Vol.133(4), (2011), p.041006-1-041006-8. 